\title{
Zum Stellenwert der neuen In-vitro-Bluttests für das Tuberkulosescreening vor Gabe von TNF- $\alpha$-Inhibitoren bei Psoriasis
}

\section{Utility of the New In-Vitro Blood Tests in Screening for Tuberculosis Prior to the Initiation of TNF- $\alpha$-Inhibitors for the Treatment of Psoriasis}

\author{
Autoren \\ R. Diel ${ }^{1}$, S. Gadola \\ Institute \\ Institut für Medizinische Soziologie, Heinrich-Heine-Universität Düsseldorf \\ School of Medicine, Department Infection, Inflammation and Repair, University of Southampton, UK
}

\section{Bibliografie}

DOI $10.1055 / \mathrm{s}-2008-1077358$

Akt Dermatol 2008; 34:

231-235 @ Georg Thieme

Verlag KG Stuttgart · New York ISSN 0340-2541

Korrespondenzadresse

\section{PD Dr. Roland Diel}

Institut für Medizinische

Soziologie

Heinrich-Heine-Universität

Düsseldorf

Postfach 101007

40001 Düsseldorf

Roland.Diel@

uni-duesseldorf.de

\section{Zusammenfassung \\ $\nabla$}

Aufgrund des erhöhten Tuberkuloserisikos beim Einsatz von TNF- $\alpha$-Inhibitoren bei der Behandlung der Psoriasis vulgaris bzw. der Psoriasisarthritis sollte bei allen Patienten eine aktive TB ausgeschlossen werden und ein Screening auf Vorliegen einer latenten TB (LTBI) vor TNF- $\alpha$-Therapie erfolgen. Das Screening sollte eine RöntgenThorax-Aufnahme, eine sorgfältigen Anamnese

Die Tumor-Nekrose-Faktor (TNF)- $\alpha$-Inhibitoren Infliximab, Etanercept und Adalimumab haben sich hervorragend in der Behandlung chronischentzündlicher Erkrankungen, insbesondere des M. Crohn, der rheumatoiden Arthritis (RA), der Spondylarthopathien und der Psoriasis bewährt. Allerdings kann eine TNF- $\alpha$-Inhibitor-Therapie die Reaktivierung einer latenten Infektion mit M. tuberculosis (LTBI) begünstigen. Ihr Einsatz ist somit mit einem steigenden TB-Risiko verbunden.

Experimentelle Studien an Mäusen haben die Bedeutung des TNF-alpha, eines pleiotropen Zytokins, als Schlüsselkomponente in der Bildung der protektiven Granulomformation bei Infektion mit M. tuberculosis (MTB) aufgezeigt. Allerdings werden MTB im Granulom lediglich in Schach gehalten, aber nicht abgetötet [1].

Aus dem Einsatz der anti-TNF-monoklonalen Antikörper ( $\mathrm{mAB}$ ) Infliximab and Adalimumab ist bekannt, dass u.a. durch direkte Neutralisierung des TNF- $\alpha$ die Granulomarchitektur zerstört werden kann. Etanercept, ein lösliches TNF- $\alpha$-Rezeptor-Fusionsprotein, das aus 2 extrazellulären Domänen des humanen p75-TNF- $\alpha$-Rezeptors und einem Fc-Fragment des humanen Immunglobulin G1 besteht, soll die Integrität des TBGranuloms in geringerem Ausmaß beeinträchtigen als die monoklonalen Antikörper Infliximab und Adalimumab. Als möglicher Grund hierfür und die Durchführung eines hochspezifischen Interferon-Gamma Release Assays (IGRA) umfassen. Da sowohl falsch positive als auch falsch negative Tuberkulinhauttest (THT)-Ergebnisse in diesen Patientengruppen erwartet werden können, wird der früher häufig durchgeführte THT nicht mehr empfohlen. Die Behandlung der LTBI bei positivem IGRA umfasst grundsätzlich eine Chemoprävention mit Isoniazid (INH) über 9 Monate.

wird die fehlende Aktivierung des Komplementsystems durch Etanercept und die damit geringere Toxizität dieser Substanz für TNF- $\alpha$-exprimierende Immunzellen vermutet [2].

Bei RA-Patienten führte in den USA der Einsatz von Infliximab zu einem mindestens 8-fach höheren Risiko, an einer TB zu erkranken als bei unbehandelten RA-Patienten (Inzidenz 52,2 vs. 6,2/100000 [3]). Die Reaktivierungsgefahr beim Einsatz von Etanercept ist offensichtlich zwar geringer als unter Infliximab (Inzidenz 28 vs. $54 / 100000$ ), beträgt gegenüber dem o.a. Basisrisiko unbehandelter RA-Patienten aber immer noch mindestens das Vierfache [2], wobei prospektive kontrollierte Vergleichsstudien bislang jedoch fehlen. Die meisten Fälle treten für gewöhnlich in der frühen Phase der Behandlung auf [4]; es wurden jedoch auch Fälle erst nach 2 Jahren berichtet [5].

In den AWMF-Leitlinien der Deutschen Dermatologischen Gesellschaft (DDG) und des Berufsverbandes Deutscher Dermatologen (BVDD) vom November 2004 [6] wird Etanercept zur Behandlung der Psoriasis-Arthritis und der mittelschweren bzw. schweren Psoriasis empfohlen, falls eine vorhergehende Basistherapie (u.a. Methotrexat oder Ciclosporin) therapeutisch unzureichend war oder wegen Komplikationen abgebrochen werden musste. Entsprechend dem Schweregrad der Erkrankung soll Etanercept als Monotherapie 
oder in Kombination mit anderen Immunsuppressiva (z.B. Methotrexat) eingesetzt werden.

Diese Richtlinien fordern auch, dass Patienten vor Beginn einer Etanercept-Therapie auf das Vorliegen einer aktiven oder latenten Tuberkulose hin untersucht werden. Zum Ausschluss einer aktiven TB soll ein Röntgen-Thorax durchgeführt werden, während zur Detektion einer LTBI bei BCG-ungeimpften Patienten zusätzlich ein Tuberkulin-Hauttest (THT) empfohlen wird. Die Richtlinien lassen offen, in welchen Fällen eine präventive Chemotherapie bei THT-Positiven gegeben werden soll.

Wie sind diese Empfehlungen, deren Überarbeitung ansteht, nach dem gegenwärtigen Stand der Tuberkulosediagnostik zu bewerten? Hierzu ist eine kurze Darstellung der gegenwärtig zur Verfügung stehenden Screeningverfahren zur Erfassung einer LTBI, nämlich des THT nach Mantoux und der seit 3 Jahren neu verfügbaren In-vitro-Bluttests, erforderlich:

\section{a) Tuberkulinhauttest:}

Diagnostisch lässt sich die verzögerte allergische Reaktion vom zellvermittelten Typ (Typ IV nach Coombs) mittels THT zur Feststellung einer latenten Tuberkuloseinfektion nutzen.

Tuberkulin ist eine teilweise gereinigte Proteinfraktion aus Überständen von Kulturen des M. tuberculosis. Nach der von Mendel und Mantoux beschriebenen Technik werden 2 Einheiten (Tuberculin Units, TU) RT 23-Tuberkulin des dänischen Statens Serum Instituts $(=0,04 \mu \mathrm{g}$ Tuberkulin PPD RT 23 SSI), entsprechend 5 TU Purified-Protein-Derivative-Standard (PPD-S), in einem Volumen von 0,1 ml mittels einer 27-G-Nadel und einer geeigneten kleinvolumigen („Tuberkulin“-) Spritze streng intradermal an der Beugeseite des Unterarms injiziert.

Bei einer vorausgegangenen Infektion entsteht eine lokalisierte Reaktion mit Erythem und Induration, die innerhalb von 24 Stunden beginnt, ihren Höhepunkt zwischen 48 und 72 Stunden erreicht und allmählich wieder über die nächsten 1-2 Wochen abklingt. Das Ablesen sollte daher vorzugsweise nach 72 Stunden und spätestens nach einer Woche erfolgen durch Messung ausschließlich der Induration (in Millimetern) in der Querachse des Unterarms.

Ein wichtiger Nachteil des THT ist die Tatsache, dass eine Infektion mit nichttuberkulösen Mykobakterien (NTM, syn: Umweltmykobakterien), deren Prävalenz eine große regionale Streubreite zeigt, aufgrund überlappender Antigene der NTM mit MTB , welche in PPD enthalten sind, ebenfalls zu einer zellulären Reaktion im THT nach Tuberkulininjektion führt. Kreuzreaktive Antigene, welche sowohl in MTB wie auch BCG vorkommen, können so auch zu einem falsch positiven Ergebnis des THT nach vorangegangener Imfung mit M. bovis (BCG) führen. Die Kreuzreaktivität bei BCG-Geimpften lässt in nur unkalkulierbarer Weise über die Zeit nach. Die Größe eines positiven THT hängt hier u.a. vom Impfstamm ab und ist bei BCG-Geimpften sehr wahrscheinlich erst bei einer Induration $>18 \mathrm{~mm}$ nicht auf eine BCG-induzierte Reaktion, sondern auf eine tuberkulöse Infektion zurückzuführen [7]. Daher kann die Interpretation des THT bei BCG-geimpften Personen nur sehr individuell erfolgen und geht auch bei nachweisbarer intensiver Exposition einer Kontaktperson meistens mit einer Unsicherheit einher.

Die Interpretation des THT-Ergebnisses in Bezug auf das Vorliegen einer möglichen LTBI hängt zum einen von testspezifischen Eigenschaften (Sensitivität und Spezifität des THT) des THT ab, zum andern aber auch von epidemiologischen Parametern, wie z.B. der Prävalenz einer Infektion mit MTB im untersuchten Kollektiv.
Die Sensitivität bezeichnet die Fähigkeit eines Testverfahrens, möglichst viele Personen mit LTBI zu erfassen und somit möglichst wenig falsch negative Ergebnisse beim Vorliegen einer MTB-Infektion zu erhalten. Die Spezifität eines Testverfahrens gibt hingegen den Prozentsatz falsch positiver Ergebnisse an. Sensitivität und Spezifität sind eng miteinander verknüpft. Aufgrund der o. a. Kreuzreaktivität von NTM, BCG und MTB ist die zu erwartende Spezifität des THT gering. Wird von Sensitivität des THT für das Vorliegen einer Infektion mit MTB gesprochen, muss eingeräumt werden, dass streng genommen nur die Sensitivität auf das aktuelle oder frühere Bestehen einer Infektion mit Mykobakterien gleich welcher Provenienz gemessen werden kann. Sensitivität und Spezifität sind beide stark abhängig vom gewählten Grenzwert (sog. Cutoff), d.h. der vorgegebenen Minimalgröße des Indurationsdurchmessers: Bei niedrigem Cutoff steigt die Wahrscheinlichkeit, viele Infizierte zu erfassen. Dafür sinkt das Unterscheidungsvermögen zwischen einer MTB-Infektion und Kreuzreaktionen mit NTM oder BCG, die oft zu geringeren Indurationsdurchmessern führen. Die offiziellen Kriterien für die Positivität des THT sind Konsensvereinbarungen von Fachgesellschaften, die durchaus unterschiedlich sein können. Während in den USA eine Cutoff von mindestens $5 \mathrm{~mm}$ gilt, um eine maximale Anzahl potentiell infizierter Personen zu erfassen, gilt in der Schweiz bei Ungeimpften ein Cutoff von $>10 \mathrm{~mm}$ und in Deutschland von $>5 \mathrm{~mm}$, wobei das spätere Erkrankungsrisiko im Allgemeinen mit dem Indurationsdurchmesser zunimmt [8].

Falsch negative THT-Ergebnisse können neben Applikationsfehlern infolge zahlreicher Ursachen für eine abgeschwächte individuelle Immunkompetenz auftreten (s. Tab. 1) und sind bei Erwachsenen prinzipiell bereits ab der 6 . Lebensdekade zu erwarten [9]. Ein wesentlicher Nachteil des THT besteht dabei darin, dass er über keine positive Testkontrolle verfügt, in welcher wie bei den Interferon-Gamma-Tests (s.u.) - prinzipiell unterschieden werden könnte, ob der negative THT-Befund auf einer möglichen Immunsuppression, einer fehlerhaften Applikation des Tuberkulins oder einer fehlenden MTB-Infektion beruhte.

Tab. 1 Mögliche Ursachen für einen falsch negativen Tuberkulinhauttest höheres Lebensalter ( $\geq 50$ Jahre) zelluläre Immundefekte (z. B. HIV-Infektion, AIDS, lymphatische Systemerkrankungen)

akute oder kurz zurückliegende schwere Virusinfektionen

(z. B. Masern, Mumps, Röteln, Influenza)

Lebendimpfungen innerhalb der letzten 6 Wochen

schwere konsumierende Erkrankungen (z. B. Malignome)

systemische Kortikoidtherapie oder Behandlung mit Immunsuppressiva fulminante tuberkulöse Erkrankung (z. B. Miliartuberkulose)

Sarkoidose

Applikationsfehler (unvollständige oder subkutane Tuberkulininjektion)

Ablesefehler (zu früh oder zu spät)

\section{b) Interferon-Gamma-Test}

(Synonym: Interferon Gamma Release Assay, IGRA): In den vergangenen Jahren gelang auf der Basis der erfolgreichen Genomsequenzierung des M. tuberculosis die Entwicklung von Testverfahren, die auf dem Nachweis von Interferon-Gamma beruhen, welches von T-Lymphozyten sezerniert wird, die im Rahmen einer aktuellen oder früheren Infektion mit M. tuberculo- 


\begin{tabular}{|c|c|c|}
\hline Name & $\begin{array}{l}\text { QuantiFERON-TB } \\
\text { Gold In-Tube }\end{array}$ & T-SPOT.TB ${ }^{\circledR}$ Test \\
\hline Hersteller & Cellestis & Oxford Immunotec \\
\hline Testmedium & Vollblut & $\begin{array}{l}\text { gereinigte mononukleäre Zellen aus } \\
\text { Venenblut (PBMC) }\end{array}$ \\
\hline Antigene & ESAT-6, CFP-10 und Tb 7.7 & ESAT-6, CFP-10 \\
\hline Messmethode & ELISA & ELISPOT \\
\hline gemessene Zielgröße & $\begin{array}{l}\text { Konzentration von sezerniertem } \\
\text { Interferon gamma/ml }\end{array}$ & $\begin{array}{l}\text { Interferon gamma-produzierende } \\
\text { T-Zellen („Spots“) pro } 250000 \text { Zellen }\end{array}$ \\
\hline benötigtes Blutvolumen & $3 \mathrm{ml}$ & $\begin{array}{l}\text { Erwachsene und Kinder } \geq 10 \text { Jahren: } 8 \mathrm{ml} \\
\text { Kinder } 2 \text { - } 9 \text { Jahre: } 4 \mathrm{ml} \\
\text { Kinder bis zu } 2 \text { Jahren: } 2 \mathrm{ml} \\
\text { Immunsupprimierte: } 16 \mathrm{ml}\end{array}$ \\
\hline Inkubation & $16-24$ Std. bei $37^{\circ} \mathrm{C}$ & $16-20$ Std. bei $37^{\circ} \mathrm{C}$ (mit $5 \% \mathrm{CO}_{2}$ ) \\
\hline $\begin{array}{l}\text { Zeitfenster nach Blut- } \\
\text { entnahme bis zur Weiter- } \\
\text { verarbeitung im Labor }\end{array}$ & $\begin{array}{l}\text { Lagerung der Blutentnahmeröhrchen } \\
\text { bis zu } 16 \text { Stunden nach Blutentnahme } \\
\text { bei Raumtemperatur vor der Inkubation }\end{array}$ & max. 8 Stunden bei Raumtemperatur \\
\hline $\begin{array}{l}\text { Test gilt als positiv, } \\
\text { wenn }\end{array}$ & $\begin{array}{l}\geq 0,35 \mathrm{IU} / \mathrm{ml} \text { (Konzentration Interferon } \\
\text { gamma) }\end{array}$ & Zahl der „Spots“ $\geq 6$ pro 250000 Zellen \\
\hline $\begin{array}{l}\text { Test gilt als nicht } \\
\text { interpretierbar } \\
\text { (,indeterminant“), } \\
\text { wenn }\end{array}$ & $\begin{array}{l}\text { (pos. Kontrolle - neg. Kontrolle } \\
<0,5 \mathrm{IU} / \mathrm{ml} \text { ) und (TB-Antigen - } \\
\text { neg. Kontrolle }<0,35 \mathrm{IU} / \mathrm{ml} \text { ) } \\
\text { oder } \\
\text { (pos. Kontrolle - neg. Kontrolle } \\
<0,5 \mathrm{IU} / \mathrm{ml} \text { ) und Differenz zwischen } \\
\text { TB-Antigen und neg. Kontrolle }<25 \% \\
\text { oder } \\
\text { neg. Kontrolle }>8,0 \mathrm{IU} / \mathrm{ml}\end{array}$ & $\begin{array}{l}\text { pos. Kontrolle }<20 \text { Spots } \\
\text { oder } \\
\text { neg. Kontrolle }>20 \text { Spots }\end{array}$ \\
\hline
\end{tabular}

Tab. 2 Vergleich der kommerziellen IGRA-Testverfahren, Quelle s. [14]
sis-Bakterien (MTB) sensibilisiert wurden. Diese Zellen werden in vitro mit spezifischen M. tuberculosis-Peptiden (ESAT-6, CFP-10 und TB7.7) stimuliert, welche bei M. bovis BCG und den meisten nichttuberkulösen Mykobakterien (NTM) (außer M. kansasii, M. szulgai und M. marinum) fehlen. Die beiden in Deutschland kommerziell erhältlichen IGRA-Tests basieren auf der direkten Messung der Interferon-Gamma-Konzentration im Vollblut (QuantiFERON-TB ${ }^{\circledR}$ Gold In-Tube, Fa. Cellestis, Australien) bzw. der Bestimmung der Zahl Interferon-Gamma sezernierender T-Lymphozyten aus isolierten peripheren mononukleären Zellen (PBMC) (T-SPOT.TB ${ }^{\circledR}$, Fa. Oxford-Immunotec, Großbritannien). Ein Vergleich der Verfahren ist in $\odot$ Tab. 2 zusammengefasst.

Beide IGRA-Tests haben bei immunkompetenten Personen eine im Vergleich zum THT mindestens gleichhohe Sensitivität. Weil ein Referenztest als „Goldstandard“ für die Diagnose der latenten tuberkulösen Infektion nicht existiert, beziehen sich die in den Publikationen veröffentlichten Sensitivitätswerte - je nach verwendetem Testsystem - von bis zu 89\% bzw. 97\% jedoch lediglich auf den Anteil IGRA-testpositiver Personen mit behandlungsbedürftiger Tuberkulose. Hieraus folgt, dass in Einzelfällen auch mit einem falsch negativen IGRA-Test gerechnet werden muss, wobei nicht vorhergesagt werden kann, ob in diesen Fällen der THT gleichfalls negativ reagieren würde oder ein diskordantes Resultat (positiver THT bei negativem IGRA) entstehen könnte.

Insbesondere bei BCG-geimpften Personen, die noch keine bekannte Exposition gegenüber Tuberkulose hatten, fand sich eine höhere Spezifität von 96-100\%, da IGRA-Tests nur die Sensibilisierung gegenüber $M$. tuberculosis und potenziell gegenüber den drei genannten NTM anzeigen. Dies bedeutet, dass anders als beim THT - keine falsch positiven Ergebnisse aufgrund einer vorherigen Impfung mit BCG und des Kontaktes mit den häufigsten NTM auftreten.
Die IGRA-Tests weisen darüber hinaus eine bessere Korrelation mit dem Ausmaß des Tuberkulosekontaktes (Nähe und Dauer) als der THT auf $[9,10]$. Matulis et al. zeigten in ihrer prospektiven Studie mit 142 rheumatischen Patienten, dass die positiven QFTResultate 5-mal häufiger mit dem Vorhandensein von Risikofaktoren für eine LTBI asssoziiert waren als ein positiver THT [12]. Applikationsfehler und Hautalterationen wie beim THT werden beim IGRA vermieden, und der Proband muss nur einmal für die Durchführung des Tests erscheinen. Darüber hinaus tritt kein Booster-Effekt auf, d.h. der Test kann beliebig wiederholt werden, und die Interpretation ist objektiv und unabhängig vom Ableser.

Allerdings bedürfen IGRA-Tests eines qualitätsgesicherten Labors, und die Vorgaben über Blutentnahme und Transport erfordern eine erprobte Logistik (Entnahme von Venenblut in speziellen Röhrchen, rascher und kältegeschützter Transport ins Labor). Bei Kindern im Vorschulalter kann die Entnahme von Venenblut Probleme aufwerfen (QuantiFERON: $3 \times 1 \mathrm{ml}$; T-SPOT.TB: $1 \times 8 \mathrm{ml})$.

IGRA-Tests können in seltenen Fällen nicht interpretierbar sein (sog. „indeterminate results“) und falsch negative Ergebnisse aufweisen, wenn die Probe keine lebenden oder stimulierbaren T-Lymphozyten enthält. Dies kann Folge eines technischen Laborfehlers (z. B. Einlagerung im Kühlschrank oder Einfrieren vor Inkubation mit resultierender Zellanergie) oder unsachgemäßen Transports sein, aber auch bei schwerer Lymphopenie und Immunsuppression vorkommen.

Bei einem nicht interpretierbaren IGRA-Test, bei dem Personen nicht auf die positive Mitogenkontrolle (Stimulierbarkeit nichtanergischer Lymphozyten durch Phytohämagglutinin) reagieren, sollte daher zunächst der Test wiederholt werden, um eine ordnungsgemäße Ausführung sicherzustellen. Bleibt das Testergebnis erneut „unschlüssig“, ist ein Immundefekt in Betracht 
zu ziehen, sodass das Vorliegen einer MTB-Infektion auf diese Weise nicht beurteilt werden kann [11].

Wie beim THT kann mit IGRA-Tests weder zwischen einer frischen und einer schon länger bestehenden Infektion noch zwischen einer LTBI und einer aktiven Tuberkulose unterschieden werden. Der exakte Zeitpunkt der frühestmöglichen Nachweisbarkeit von IGRA ist studienmäßig nicht belegt, dürfte aber dem zeitlichen Ablauf der mit Tuberkulin messbaren Konversion (2-8 Wochen) entsprechen.

Das Risiko, nach frischer MTB-Infektion einmal im Laufe des Lebens an aktiver TB zu erkranken, wurde aufgrund von THT-basierten Reihenuntersuchungen auf maximal 10\% geschätzt. Aufgrund der schlechten Spezifität des THT dürfte dieses Risiko deutlich höher liegen. Hierauf weist eine jüngere Kohortensstudie bei engen Kontaktpersonen hin, bei der eine Progressionsrate der QFT-Positiven (IGRA-positiven) von 14,6\% bereits im Zeitraum von 2 Jahren gemessen wurde [14]. Bereits hierdurch kommt dem Screening von Patienten vor dem Einsatz von TNF$\alpha$-Blockern durch IGRA-Tests eine besondere Bedeutung zu. Obgleich die Sensitivität der IGRA-Tests bei immunsupprimierten Patienten derjenigen des THT offensichtlich überlegen ist, ist sie jedoch noch nicht definitiv bestimmt, sodass ein negatives Testergebnis unter sorgfältiger Berücksichtigung des klinischen Gesamtbildes bzw. hilfsweise unter Berücksichtigung der Expositionsintensität der Kontaktperson zum Indexfall eingeordnet werden muss.

Da Kontaktpersonen infektiöser TB-Indexpatienten in der Regel immunkompetent sind, hatte das Deutsche Zentralkomitee zur Bekämpfung der Tuberkulose (DZK) in seinen aktuellen Empfehlungen zu den Umgebuntersuchungen [15] einen vollständigen, routinemäßigen Ersatz des THT durch IGRA-Tests noch nicht empfohlen. Stattdessen wurde bei BCG-ungeimpften und kooperativen Personen unter 50 Jahren eine Kombination beider Testverfahren mit primärer Durchführung des THT angestrebt, bei der die Verknüpfung einer hohen THT-Sensitivität mit niedrigem Grenzwert (Indurationsdurchmesser $>5 \mathrm{~mm}$ ) bei einem positiven Testergebnis mit der hiervon unabhängigen hohen Spezifität eines nachfolgenden IGRA-Tests zu Synergieeffekten führen soll. Diese konditionale Anwendung des IGRA-Tests lediglich als Bestätigungstest eines vorherigen THT ist bei Personen mit chronisch-entzündlichen Immunerkrankungen nicht sinnvoll: Aufgrund der in der Regel bestehenden immunsupprimierenden Therapie mit Kortikoiden und/oder Methothrexat und unabhängig hiervon möglichen Alterationen der T-Zell-Funktion [16,17] ist bei diesem Patientengut grundsätzlich mit einem falsch negativen THT zu rechnen. Neben mehreren Fallberichten, in denen klinisch und radiologisch zuvor völlig unauffällige Patienten [18], aber auch Patienten mit den radiologischen Zeichen einer früheren TB [19] einen negativen Hauttest aufwiesen, liegen hierzu inzwischen zahlreiche Studien vor:

Raval et al. [20] analysierten die zwischen dem 1. November 2001 und 30. Mai 2006 bei der U.S. Food and Drug Administration eingegangenen 130 Spontanmeldungen von TB bei Patienten mit Infliximab-Therapie. In der Untergruppe von 67 Fällen, die zuvor einen THT erhalten hatten, waren 34 Patienten $(51 \%)$ vor Beginn der Infliximab-Therapie THT-negativ.

Ponce de Leon et al. [21] fanden in ihrer Studie mit 112 konsekutiven RA-Patienten und 96 gesunden Kontrollpersonen eine deutlich niedrigere THT-Positivität der RA-Patienten; 79 RA-Patienten $(70,6 \%)$ hatten einen negativen THT im Vergleich zu 25 (26\%) in der Kontrollgruppe.
In einer Studie von Vassilopoulos et al. [22] mit 70 Patienten aus dem rheumatischen Formenkreis waren 4 von 16 T-Spot.TB-Positiven gleichzeitig THT-negativ. $41 \%$ der 70 Patienten hatten Prednisolon (im Mittel 6,8 $\pm 5,2 \mathrm{mg}$ ) über einen mittleren Zeitraum von 26 Monaten erhalten, deren fortlaufender Einsatz mit einer THT-negativ-/T-Spot-positiv-Diskordanz assoziiert waren. Sellam et al. [23] untersuchten 13 von 68 Patienten mit chronisch-entzündlichen Erkrankungen, bei denen von 13 mit einem positiven Elispot-Test 5 (38,5\%) einen negativen THT aufwiesen. Hieraus ergibt sich, dass nach Ausschluss einer aktiven LungenTB durch Rö-Thorax und sorgfältige klinische Untersuchung bzw. Befragung nach eventuellen TB-assoziierten Symptomen primär ein IGRA-Test duchgeführt werden sollte. Sollte dieser negativ sein, erübrigt sich in der Regel eine anschließende Chemoprävention. Nur in Ausnahmefällen, wenn trotz eines negativen IGRA-Tests eine frühere enge Exposition gegenüber einem Patienten mit infektiöser Lungen-TB anamnestisch plausibel gemacht werden kann, eine BCG-Impfung unter Berücksichtigung der Impfpolitik im jeweiligen Herkunftsland des Patienten unwahrscheinlich und/oder ein IGRA-Test auch in der Wiederholung unbestimmbar ist, ist ein ergänzend durchgeführter THT möglicherweise sinnvoll und könnte dann zu einem höheren prädiktiven Werts des LTBI-Screenings als Grundlage für die Entscheidung zu einer präventiven Chemotherapie beitragen [24].

Die neuen Schweizer Konsensempfehlungen [25] zum TB-Screening vor TNF- $\alpha$-Therapie verzichten jedoch auch in diesen Einzelfällen auf den Einsatz eines THT und beschränken sich lediglich auf die Vorgeschichte des Patienten. Dieser restriktive Ansatz hilft nicht nur, die Zahl vermeidbarer TB-Fälle bei diesen vulnerablen Patienten auf ein Minimum zu reduzieren, sondern auch unnötige präventive Therapien zu vermeiden, die aufgrund ihrer Hepatotoxizität eines regelmäßigen klinischen und laborparametrischen Monitorings bedürfen. Als weitere Indikation zur Chemoprävention vor TNF- $\alpha$-Therapie werden unabhängig von einem IGRA-Test röntgenologische Zeichen einer durchgemachten, aber unbehandelten Tuberkulose ohne Anhalt für Aktivität (kalzifizierte Knötchen, Spitzenfibrose, Pleuraschwielen) betrachtet [24,25].

Die Behandlung der LTBI umfasst grundsätzlich eine Chemoprävention mit INH über 9 Monate. Der beste Zeitpunkt einer anti-TNF- $\alpha$-Therapie bei Patienten, die sich einer INH-Chemoprävention unterziehen, ist unbekannt. Eine Therapie mit TNF$\alpha$-Blockern sollte idealerweise nach Abschluss der INH-Chemoprävention oder aber, sofern der klinische Befund dies nicht zulässt, frühestens 1-2 Monate nach Einleitung der Chemoprävention begonnen werden, um abschätzen zu können, ob im fortlaufenden Monitoring die Chemoprävention gut vertragen und damit fortgeführt werden kann [4,25]. Bei guter Adhärenz ist bei Patienten mit chronisch-entzündlichen Erkrankungen mit einer Effektivität der INH-Therapie von ca. $80 \%$ zu rechnen $[26,27]$. 
Abstract

Utility of the New In-Vitro Blood Tests in Screening for Tuberculosis Prior to the Initiation of TNF- $\alpha$-Inhibitors for the Treatment of Psoriasis

$\nabla$

Due to the elevated risk of tuberculosis in patients under treatment with TNF-alpha-inhibitors for the treatment of Psoriasis vulgaris or Psoriasisarthris, all patients should undergo examination to exclude active $\mathrm{TB}$, and screening of all patients should be undertaken to detect eventual cases of latent TB (LTBI) prior to the beginning of TNF- $\alpha$ therapy. Screening should consist of a chest X-ray, a careful evaluation of risk factors and a blood test by means of a highly specific IGRA test. Because both-false positive and false-negative results must be expected when using the tuberculin skin test in this patient population, that test is no longer recommended. Treatment of LTBI in the case of a positive IGRA-Test consits, generally, of a 9-month course of chemoprevention with INH.

\section{Literatur}

1 Ehlers $S$. Why does tumor necrosis factor targeted therapy reactivate tuberculosis? J Rheumatol (Suppl) 2005; 74: 35 - 39

2 Wallis RS, Broder MS, Wong JY, Hanson ME, Beenhouwer DO. Granulomatous infectious diseases associated with tumor necrosis factor antagonists. Clin Infect Dis 2004; 38: 1261 - 1265

3 Wolfe F, Michaud K, Anderson J, Urbansky K. Tuberculosis infection in patients with rheumatoid arthritis and the effect of infliximab therapy. Arthritis Rheum 2004; 50: 372-379

4 Keane J. TNF-blocking agents and tuberculosis: new drugs illuminate an old topic. Rheumatology 2005; 44: $714-720$

5 Schiff MH, Burmester GR, Kent JM et al. Safety analyses of adalimumab (Humira) in global clinical trials and US postmarketing surveillance of patients with rheumatoid arthritis. Ann Rheum Dis 2006; 65: 889894

6 AWMF-Leitlinien-Register Nr. 013/036, Entwicklungsstufe 1. www.awmf-leitlinien.de

7 Tissot F, Zanetti G, Francioli P et al. Influence of Bacille Calmette-Guérin vaccination on size of tuberculin skin test reaction: to what size? Clin Inf Dis 2005; 40: 211 - 217

8 Horsburgh CR Jr. Priorities for the treatment of latent tuberculosis infection in the United States. N Engl J Med 2004; 350: 2060 - 2079

9 Simon K, Gutland M. Die Tuberkulinprobe in der Klinik - Dosierung und Wertung. Prax Klin Pneumol 1988; 42: 423-426

10 Diel R, Ernst M, Döscher $G$ et al. Avoiding the effect of BCG vaccination in detecting Mycobacterium tuberculosis infection with a blood test. Eur Respir J 2006; 28: 16-23

11 Diel R, Nienhaus A, Lange $C$ et al. Tuberculosis contact investigation with a new, specific blood test in a low-incidence population containing a high proportion of BCG-vaccinated persons. Respiratory Research 2006; 7: 77

12 Matulis G, Jüni P, Villiger PM, Gadola SD. Detection of latent tuberculosis in immunosuppressed patients with autoimmune diseases - per- formance of a mycobacterium tuberculosis antigen specific IFN-gamma assay. Ann Rheum Dis 2008; 67: 84 - 90. Epub 2007 Jul 20.

13 Piana F, Codecasa LR, Cavallerio P et al. Use of a T-cell based test for detection of tuberculosis infection among immunocompromised patients. Eur Respir J 2006; 28: 1 - 34

14 Diel R, Loddenkemper R, Meywald-Walter K, Nieman S, Nienhaus A. Predictive value of a whole-blood IFN- $\gamma$ assay for the development of active TB disease after recent infection with $M$. tuberculosis. AJRCCM 2008; 177: 1164-1170. Epub 2008 Feb 14

15 Diel R, Forssbohm M, Loytved G et al German Central Committee against Tuberculosis. Empfehlungen für die Umgebungsuntersuchungen bei Tuberkulose [Recommendations for environmental contact tracing in tuberculosis]. Pneumologie 2007; 58: 657-665

16 Panayi G, Corrigall V, Pitzalis C. Pathogenesis of rheumatoid arthritis. The role of T cells and others beasts. Rheum Dis Clin North Am 2001; 27: $1-18$

17 Mow WS, Abreu-Martin MT, Papadakis KA et al. High incidence of anergy in inflammatory bowel disease patients limits the usefulness of PPD screening before infliximab therapy. Clin Gastroenterol Hepatol 2004; 2: $309-313$

18 Malipeddi AS, Rajendran R, Kallarackal G. Disseminated tuberculosis after anti-TNF $\alpha$ treatment. Lancet 2007; 369: 162

19 Efthimiou P, Sood S. Quantiferon TB gold test: The new standard for screening of latent tuberculosis in patients with rheumatoid arthritis? Ann Rheum Dis 2007; 66: 276. Epub 2006 Nov 24.

20 Raval A, Akhavan-Toyserkani G, Brinker A, Avigan M. Brief Communication: Characteristics of Spontaneous Cases of Tuberculosis Associated with Infliximab. Ann Intern Med. 2007; 147: 699 - 702

21 Ponce de Leon D, Acevedo-Vasquez E, Alvizuri S, Gutierrez C, Cucho M, Alfaro J, Perich R, Sanchez-Torres A, Pastor C, Sanchez-Schwartz C, Medina M, Gamboa R, Ugarte M. Attenuated response to purified protein derivative in patients with rheumatoid arthritis: study in a population with a high prevalence of tuberculosis. Ann Rheum Dis 2005; 64: $1360-1361$

22 Vassilopoulos D, Stamoulis N, Hadziyannis E, Archimandritis A. Usefulness of Enzyme-Linked Immunospot Assay (Elispot) Compared to Tuberculin Skin Testing for Latent Tuberculosis Screening in Rheumatic Patients Scheduled for Anti-Tumor Necrosis Factor Treatment. J Rheumatol 2008 Mar 15; [Epub ahead of print]

23 Sellam J, Hamdi H, Roy C, Baron G, Lemann M, Puéchal X, Breban M, Berenbaum F, Humbert M, Weldingh K, Salmon D, Ravaud P, Emilie D, Mariette X RATIO (Research Axed on Tolerance of Biotherapies) Study Group. Comparison of in vitro-specific blood tests with tuberculin skin test for diagnosis of latent tuberculosis before anti-TNF therapy. Ann Rheum Dis 2007; 66: 1610 - 1615

24 Gardam MA, Keystone EC, Menzies R et al. Anti-tumour necrosis factor agents and tuberculosis risk: mechanisms of action and clinical management. Lancet Infect Dis 2003; 3: 148-55

25 Beglinger C, Dudler J, Mottet C, Nicod L, Seibold F, Villiger PM, Zellweger JP. Screening for tuberculosis infection before initiation of anti-TNF- $\alpha$ therapy. Swiss Med Wkly 2007; 137: 621 - 622

26 Sichletidis L, Settas L, Spyratos D, Chloros D, Patakas D. Tuberculosis in patients receiving anti-TNF agents despite chemoprophylaxis. Int J Tuberc Lung Dis 2006; 10: 1127 - 1132

27 Carmona L, Gómez-Reino JJ, Rodríguez-Valverde V, Montero D, PascualGómez E, Mola EM, Carreño L, Figueroa M BIOBADASER Group. Effectiveness of recommendations to prevent reactivation of latent tuberculosis infection in patients treated with tumor necrosis factor antagonists. Arthritis Rheum 2005; 52: 766-772 